\title{
Locoregionally advanced nasopharyngeal carcinoma treated with intensity-modulated radiotherapy plus concurrent weekly cisplatin with or without neoadjuvant chemotherapy
}

\author{
Chan Woo Wee, MD', Bhumsuk Keam, MD, PhD 2,3 , Dae Seog Heo, MD, PhD², \\ Myung-Whun Sung, MD, PhD ${ }^{4}$, Tae-Bin Won, MD, PhD ${ }^{4}$, Hong-Gyun Wu, MD, PhD 1,3,5 \\ Department of ${ }^{1}$ Radiation Oncology and ${ }^{2}$ Internal Medicine, Seoul National University College of Medicine, Seoul; \\ ${ }^{3}$ Cancer Research Institute, Seoul National University College of Medicine, Seoul; ${ }^{4}$ Department of Otorhinolaryngology-Head \\ and Neck Surgery, Seoul National University College of Medicine, Seoul; ${ }^{5}$ Radiation Research Institute, \\ Medical Research Center, Seoul National University, Seoul, Korea
}

Purpose: The outcomes of locoregionally advanced nasopharyngeal carcinoma patients treated with concurrent chemoradiation (CCRT) using intensity-modulated radiotherapy (IMRT) with/without neoadjuvant chemotherapy (NCT) were evaluated.

Materials and Methods: Eighty-three patients who underwent NCT followed by CCRT (49\%) or CCRT with/without adjuvant chemotherapy (51\%) were reviewed. To the gross tumor, 67.5 Gy was prescribed. Weekly cisplatin was used as concurrent chemotherapy.

Results: With a median follow-up of 49.4 months, the 5-year local control, regional control, distant metastasis-free survival (DMFS), disease-free survival (DFS), and overall survival rates were 94.7\%, 89.3\%, 77.8\%, 68.0\%, and 81.8\%, respectively. In multivariate analysis, the American Joint Committee on Cancer stage $(p=0.016)$ and $N$ stage $(p=0.001)$ were negative factors for DMFS and DFS, respectively. Overall, NCT demonstrated no benefit and an increased risk of severe hematologic toxicity. However, compared to patients treated with CCRT alone, NCT showed potential of improving DMFS in stage IV patients.

Conclusion: CCRT using IMRT resulted in excellent local control and survival outcome. Without evidence of survival benefit from phase III randomized trials, NCT should be carefully administered in locoregionally advanced nasopharyngeal carcinoma patients who are at high-risk of developing distant metastasis and radiotherapy-related mucositis. The results of ongoing trials are awaited.

Keywords: Nasopharyngeal carcinoma, Intensity-modulated radiotherapy, Concurrent chemoradiotherapy, Induction chemotherapy

\section{Introduction}

While nasopharyngeal carcinoma (NPC) is a rare malignancy in most parts of the world, it is one of the most common malignancies in endemic areas, such as southern China,
Southeast Asia, the Middle East, North Africa, Alaska, Greenland, and the Mediterranean. In these areas, it is strongly associated with Epstein-Barr virus infection and differs pathologically and clinically from other cancers of the head and neck (HEN) $[1,2]$. While surgery is still the mainstay of

Received 24 March 2015, Revised 21 May 2015, Accepted 1 June 2015.

Correspondence: Hong-Gyun Wu, MD, PhD, Department of Radiation Oncology, Seoul National University Hospital, 101 Daehak-ro Jongno-gu, Seoul 110-744, Korea. Tel: +82-2-2072-3177, Fax: +82-2-742-2073, E-mail: wuhg@snu.ac.kr

(c) This is an Open Access article distributed under the terms of the Creative Commons Attribution Non-Commercial License (http://creativecommons.org/ licenses/by-nc/4.0/) which permits unrestricted non-commercial use, distribution, and reproduction in any medium, provided the original work is properly cited.

www.e-roj.org 


\section{ROJ Radiation Oncology Journal}

other HEtN cancers, radiotherapy (RT) is the backbone of treatment for NPC. Despite its sensitivity to RT, the survival of patients with locoregionally advanced NPC (LA-NPC; stage IIIIVB by the American Joint Committee on Cancer [AJCC] staging system 7 th edition) is poor, with less than $60 \%$ surviving at 5 years when treated by RT alone $[3,4]$.

The Intergroup 0099 study [5] demonstrated absolute survival benefit with platinum-based concurrent chemoradiation (CCRT) plus adjuvant chemotherapy (ACT) compared to RT alone. Multiple phase III randomized trials [6-10] over the past 15 years have established CCRT as the standard approach in LANPC. However, CCRT was followed by ACT in some studies [57], whereas chemotherapy was given only during the course of RT in others [8-10]. Therefore, debates on the necessity of the addition of ACT to CCRT still exist. Since ACT was part of the landmark Intergroup 0099 study, it was often adopted for clinical trial protocols [5-7]. However, it has failed to demonstrate survival benefit over CCRT alone [11]. Moreover, while platinum-based concurrent chemotherapy was adopted in majority of the trials, the schedule varied from weekly to triweekly administration. The total RT dose varied from 62.5 to 74 Gy and dose per fraction, from 1.8 to 2.5 Gy per fraction. None of the trials required intensity-modulated radiotherapy (IMRT), which is considered the 'standard' or 'preferred' technique in the current National Comprehensive Cancer Network guidelines [12]. The necessity of additional ACT, optimal concurrent chemotherapy schedule, and optimal RT dose-fractionation scheme in patients treated with CCRT remain to be defined.

The role of neoadjuvant chemotherapy (NCT) in addition to CCRT is also controversial. NCT is often administered prior to CCRT in clinics to debulk the primary tumor and eradicate the micrometastatic tumor burden. However, there is a lack of evidence of a definite overall survival (OS) benefit over CCRT alone [13-17]. The results from the only two phase II randomized trials $[18,19]$ conflict with each other. Four randomized phase III trials (NCT00201396, NCT00997906, NCT00828386, and NCT01245959) are ongoing to solve this conflict.

Therefore, we evaluated the role of NCT and ACT in combination with CCRT using IMRT only. We also assessed the feasibility and survival outcomes of a homogenous CCRT regimen in use for over ten years at our institution.

\section{Materials and Methods}

\section{Ethics}

This study was performed with the approval from the Health

\section{CCRT with IMRT in stage III-IV nasopharyngeal carcinoma}

Institutional Review Board of the Seoul National University on June 13, 2013.

\section{Patient eligibility}

Medical records of the eligible patients were retrospectively reviewed. Patients were eligible if they had biopsy-proven, previously untreated, and stage III-IVB NPC (by the AJCC staging system 7th edition) treated with curative aim by IMRT concurrently with weekly intravenous cisplatin with or without NCT or ACT. Other eligibility criteria included assessable disease, at least 18 years of age, Eastern Cooperative Oncology Group performance score of 0 to 1 , adequate baseline bone marrow reserve, renal, and hepatic function to undergo optimal chemotherapy, no previous history of malignancies within 5 years prior and after the diagnosis of NPC, and no previous history of RT or chemotherapy. All patients should have undergone at least one of either computed tomography (CT) or magnetic resonance imaging (MRI) of the HEtN region at the time of diagnosis. Patients who underwent systemic workup by chest $\mathrm{X}$-ray, chest $\mathrm{CT}$, or positron emission tomographycomputed tomography were included.

\section{Radiotherapy}

For CT simulation, all patients were fixed in the supine position by thermoplastic masks with the neck slightly extended. Simulations were done in $3 \mathrm{~mm}$ slices using intravenous contrast media. Scans were performed from the vertex to at least $3 \mathrm{~cm}$ below the clavicular head. MRI-CT fusions were not routinely used.

The gross tumor volume (GTV) of the primary site and neck included all disease visualized on either CT or MRI, as well as suspicious areas on physical and endoscopic exams. The high-risk clinical target volume (CTV) was defined using three-dimensional auto-expansion of $5 \mathrm{~mm}$ around the GTV and modifying it regarding anatomical architecture. The intermediate-risk CTV included the high-risk CTV with an additional 5-mm margin plus bilateral retropharyngeal nodes and involved cervical nodal stations with or without one subsequent uninvolved cervical nodal station, depending on the physician's decision. The intermediate-risk CTV also encompassed the entire nasopharyngeal mucosa and suspicious areas at risk among the skull base, parapharyngeal spaces, inferior sphenoid sinuses, posterior nasal cavity, posterior maxillary sinuses, and the cavernous sinuses. The low-risk CTV included bilateral cervical nodes not covered by the high- or intermediate-risk CTV (Fig. 1). Level IA and IB cervical nodal areas were not included in any CTV unless there 
A
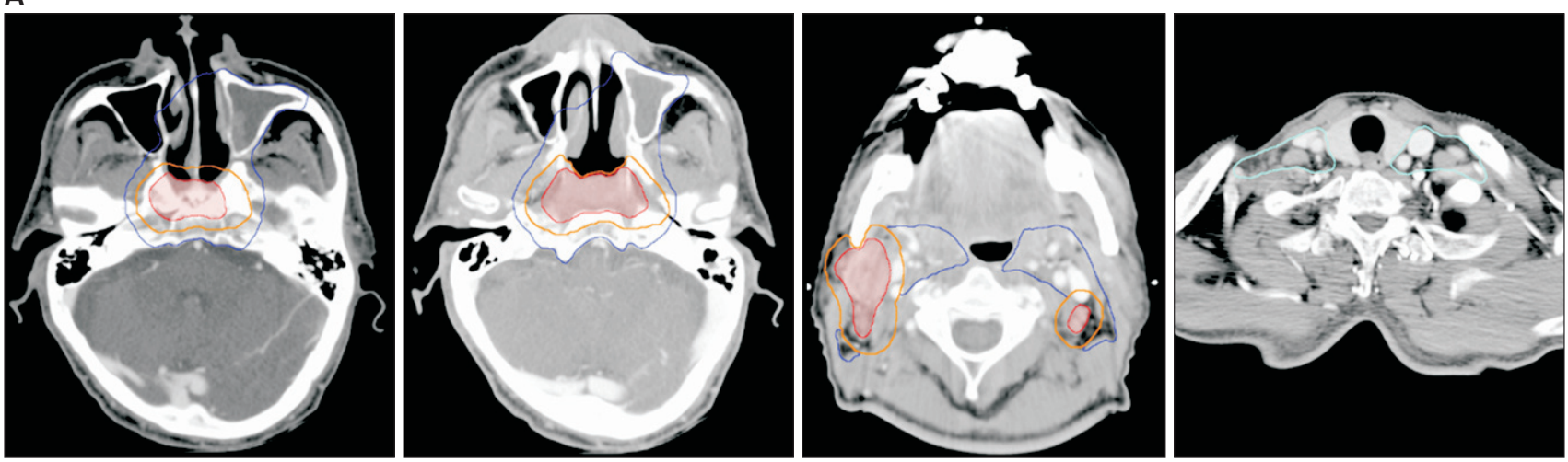

B
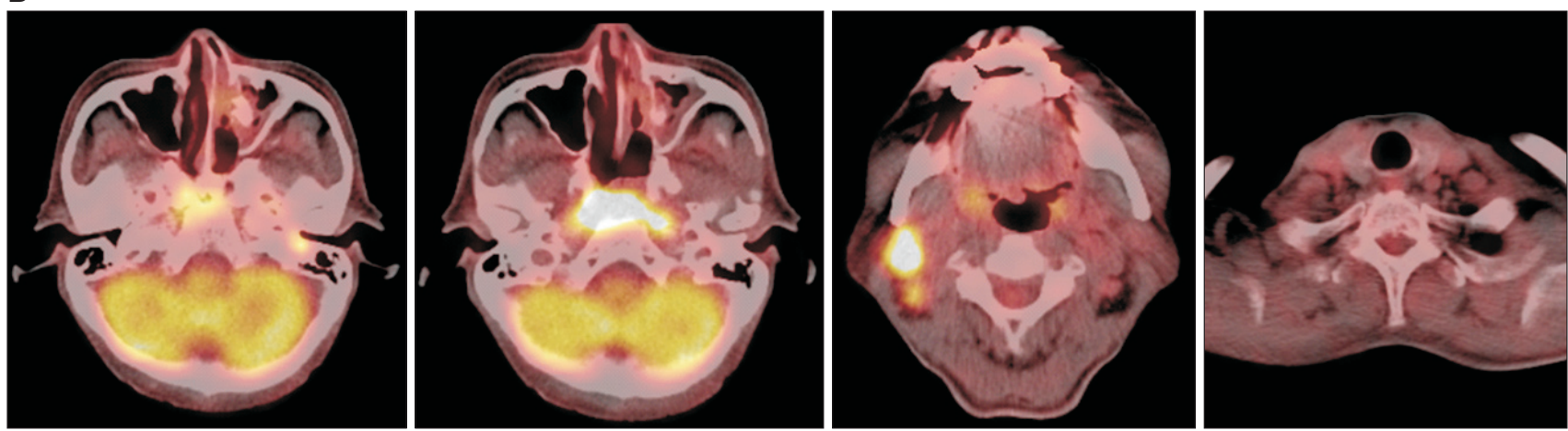

Fig. 1. An example of target volume delineation for intensity-modulated radiotherapy. A 61-year-old male had a primary lesion involving the bilateral nasopharyngeal mucosa and central skull base, and multiple clinically involved lymph nodes at bilateral level II and right level III (clinical stage T3N2 according to the American Joint Committee on Cancer staging system 7th edition). Level IV and V cervical chains were not involved. (A) The high-risk clinical tumor volume (CTV, orange line) is created by 5-mm auto-expansion margin around the gross tumor volume (red line). The intermediate-risk CTV (blue line) is further expanded with a 5-mm margin around the high-risk CTV of the primary lesion, encompassing the involved cervical nodal stations and areas of high-risk. Bilateral retropharyngeal nodes and the entire nasopharyngeal mucosa are routinely included in the intermediate-risk CTV. The low-risk CTV (cyan line) includes the remnant cervical lymph nodes. (B) Images of fluorodeoxyglucose positron emission tomography taken before concurrent chemoradiation at a similar level to the displayed target volumes.

was clinical evidence of involvement. The high-, intermediate-, and low-risk PTVs were created by $3 \mathrm{~mm}$ auto-expansion (except one patient with $2 \mathrm{~mm}$ auto-expansion) of the high-, intermediate-, and low-risk CTVs, respectively, and were restricted to the body. Doses of 67.5 Gy, 54-60 Gy, and $48 \mathrm{~Gy}$ in 30 daily fractions were applied to the high-, intermediate-, and low-risk PTVs, respectively.

Organs-at-risk (OARs) including the eyeballs or lenses, optic nerves or chiasm, brain stem, spinal cord, pituitary gland, temporomandibular joints, middle or inner ears, submandibular glands, and parotid glands were contoured for every patient. Dose restriction to the OARs at plan evaluation satisfied the Radiation Therapy Oncology Group (RTOG) protocol for IMRT [20].

Inverse treatment planning using simultaneous integrated boost technique was performed using the Eclipse (Varian Medical Systems, Palo Alto, CA, USA) treatment planning software system. Six-megavoltage photon beams were used for arrangement in all cases. Eighty patients were treated with step-and-shoot or sliding window IMRT. Three patients were treated with volumetric modulated arc therapy.

\section{Chemotherapy}

During the course of CCRT, all patients were to receive intravenous administration of cisplatin at $35 \mathrm{mg} / \mathrm{m}^{2}$ per week starting on the day of RT initiation. Patients who have not completed their planned RT or concurrent chemotherapy will be described later. The decision to use NCT prior to CCRT was made either by the medical oncologist before referral of the patient to the radiation oncologist, or by the multidisciplinary 
team after a face-to-face discussion between the radiation oncologists, medical oncologists, and HEtN surgeons. At the multidisciplinary round, the use of NCT was frequently chosen when the parotid gland was unlikely to be spared or severe mucositis could not be avoided due to bulky tumor burden. For patients treated with NCT, a docetaxel/5-fluorouracil/cisplatin combination was the most commonly used regimen, followed by docetaxel/cisplatin and 5 -fluorouracil/cisplatin. For all patients treated with ACT, 5-fluorouracil/cisplatin was given as described in the Intergroup 0099 trial [5]. Details of NCT and ACT regimens are shown on Table 1.

\section{Follow-up}

All patients were followed up on a weekly basis during CCRT. After completing the initial treatment course, patients were examined by at least two or more of their attending radiation oncologist, HEtN surgeon, or medical oncologist at threemonth intervals for the first one or two years and at sixmonth intervals for the following one to three years. Followup consisted of imaging, physical examination, endoscopic examinations, and laboratory tests. In case of suspicious disease progression or major toxicity, the follow-up intervals were shortened.

Acute and late RT-related toxicities were evaluated according to the RTOG/European Organisation for Research and Treatment of Cancer radiation morbidity scoring criteria. Acute hematologic toxicities were evaluated according to the National Cancer Institute (NCI) Common Toxicity Criteria ver. 3.0.

\section{Statistical analysis}

All events were measured from the date of commencement of any definitive treatment. The local control (LC), regional control $(R C)$, distant metastasis-free survival (DMFS), disease-free survival (DFS), and OS rates were estimated with the KaplanMeier method. The level of significance was set at a twotailed $p$-value of $<0.05$. To detect the differences in actuarial rates among different prognostic factors, the log-rank test was performed for univariate analyses. Multivariate analyses using the Cox proportional hazard model with a backward stepwise method were performed to test the independent significance of different prognostic factors. For multivariate analyses, factors were included if the $p$-values were lower than 0.1 in a univariate analysis. The chi-squared test was used to compare adverse events and other categorical variables between treatment arms. All analyses were conducted using the Statistical Package for the Social Science software ver. 18.0 (SPSS Inc., Chicago, IL, USA).

\section{Results}

\section{Patients}

From December 2004 to October 2012, 83 LA-NPC patients were treated by IMRT concurrently with weekly intravenous cisplatin at our institution. The median age at diagnosis was 49 years. There was a strong bias for males and World Health Organization (WHO) type Ilb. More than half the patients had a high T stage disease (T1-T2, 42.2\%; T3-T4, 57.8\%). Most

Table 1. Neoadjuvant and adjuvant chemotherapy regimens

\begin{tabular}{|c|c|c|}
\hline Regimen & Dosage & Cycles \\
\hline \multicolumn{3}{|c|}{ Neoadjuvant chemotherapy $(n=41)$} \\
\hline \multicolumn{3}{|l|}{ DFP } \\
\hline Docetaxel $(n=24)$ & $70 \mathrm{mg} / \mathrm{m}^{2}$ on day 1 & 3 Cycles in 9 weeks \\
\hline 5-Fluorouracil & $1,200 \mathrm{mg} / \mathrm{m}^{2}$ on days $1-3$ & \\
\hline Cisplatin & $40 \mathrm{mg} / \mathrm{m}^{2}$ on day 1 & \\
\hline \multicolumn{3}{|l|}{$\mathrm{DP}$} \\
\hline Docetaxel $(n=12)$ & $75 \mathrm{mg} / \mathrm{m}^{2}$ on day 1 & 3 Cycles in 9 weeks ${ }^{a)}$ \\
\hline Cisplatin & $75 \mathrm{mg} / \mathrm{m}^{2}$ on day 1 & \\
\hline \multicolumn{3}{|l|}{$\mathrm{FP}$} \\
\hline 5-Fluorouracil $(n=5)$ & $1,000 \mathrm{mg} / \mathrm{m}^{2}$ on days $1-5$ & 3 Cycles in 9 weeks $^{\text {a) }}$ \\
\hline Cisplatin & $100 \mathrm{mg} / \mathrm{m}^{2}$ on day 1 & \\
\hline \multicolumn{3}{|c|}{ Adjuvant chemotherapy $(n=12)$} \\
\hline \multicolumn{3}{|c|}{ FP } \\
\hline 5-Fluorouracil $(n=12)$ & $1,200 \mathrm{mg} / \mathrm{m}^{2}$ on days $1-4$ & 3 Cycles in 12 weeks $^{b)}$ \\
\hline Cisplatin & $40 \mathrm{mg} / \mathrm{m}^{2}$ on day $1-2$ & \\
\hline
\end{tabular}

DFP, docetaxel/5-fluorouracil/cisplatin combination; DP, docetaxel/cisplatin combination; FP, 5-fluorouracil/cisplatin combination.

${ }^{a)}$ One patient received 2 cycles in 6 weeks. ${ }^{b}$ One patient and 4 patients received 2 and 4 cycles of adjuvant chemotherapy, respectively. 
presented with cervical nodal metastases (N0, 9.6\%; N1-N2, $63.9 \%$; N3b, 26.5\%) but there were no patients with bulky nodal metastases exceeding $6 \mathrm{~cm}$ (N3a). Slightly more than half the patients (55.4\%) had non-metastatic stage IV disease. Patient demographics and clinical characteristics of the total cohort are summarized in Table 2.

\section{Treatment}

The median duration of CCRT was 42 days (range, 35 to 78 days) and 80 patients completed CCRT within 50 days. The duration of CCRT for the other three patients was 53, 58, and 78 days. The reasons of prolongation were a long holiday, severe mucositis, and psychological issues of depression and claustrophobia, respectively. Only one patient was initially planned to receive curative RT with $63 \mathrm{~Gy}$ in 28 fractions due to a remarkable response following three cycles of NCT. Of the remaining 82 patients, two did not complete their planned RT of 67.5 Gy. Without any definite severe toxicity, the two patients refused further treatment after 63 Gy in 28 fractions and 65.25 Gy in 29 fractions. The planned concurrent chemotherapy was six cycles of weekly intravenous cisplatin in all patients. The actual median number of applied cycles was also six (range, 2 to 7). Eleven patients received incomplete concurrent chemotherapy due to patient refusal of chemotherapy (two patients), azotemia (one patient), grade 3 nausea (two patients), grade 3 mucositis (four patients), grade

Table 2. Patient characteristics of the whole cohort

\begin{tabular}{lc}
\hline Characteristic & Value \\
\hline Age (yr) & $49(21-77)$ \\
Sex & $57(68.7)$ \\
Male & $26(31.3)$ \\
Female & \\
Histology & $25(30.1)$ \\
WHO I-Ila & $58(69.9)$ \\
WHO Ilb & \\
T classification & $35(42.2)$ \\
T1-T2 & $48(57.8)$ \\
T3-T4 & $8(9.6)$ \\
N classification & $53(63.9)$ \\
NO & $22(26.5)$ \\
N1-N2 & \\
N3b & $37(44.6)$ \\
Stage group & $46(55.4)$ \\
III & \\
IVA-IVB &
\end{tabular}

Values are presented as median (range) or number of patients (\%). WHO, World Health Organization.
4 thrombocytopenia (one patient), and drug hypersensitivity (one patient). A median of three cycles was administered for both the 41 patients who received NCT and 12 patients who received $A C T$.

\section{Survival and disease control}

With a median follow-up of 49.4 months (range, 6.0 to 123.7 months) for survivors, the 5-year LC, RC, DMFS, DFS, and OS rates were $94.7 \%, 89.3 \%, 77.8 \%, 68.0 \%$, and $81.8 \%$, respectively. Of the 83 patients, 18 died, 3 failed locally, 8 failed regionally, and 16 failed systemically. The most common site of distant failure was bone, followed by liver, mediastinum, and lung.

\section{Prognostic factors}

To adjust for prognostic factors, the following parameters were included in the analysis: age ( $<50$ vs. $\geq 50$ ), sex, histology (WHO type Ilb vs. others), T stage (1-2 vs. $3-4), N$ stage (0 vs. 1-2 vs. 3b), AJCC stage (III vs. IV), use of NCT, and use of any additional chemotherapy (either NCT or ACT) other than concurrent administration with RT. In a univariate analysis (Table 3), there were no significant prognostic factors for OS. N stage was a significant factor for RC ( $p=0.032)$, DMFS $(p=0.005)$, and DFS $(p<0.001)$ rates. AJCC stage IV was also a significantly poor prognostic factor for DMFS rate $(p=0.006)$ and DFS $(p=$ 0.009) compared to stage III. The use of NCT or chemotherapy in addition to CCRT did not show clinical benefit with any endpoint.

In a multivariate analysis (results not shown), stage IV disease was proved to be an independent adverse factor for DMFS ( $p=0.016$, hazard ratio $=6.18$ ) compared to stage III disease. Advanced $\mathrm{N}$ stage was an adverse factor for DFS ( $p=$ 0.001).

\section{Toxicity}

No grade 5 toxicity occurred in the entire cohort (Table 4). During the course of NCT, CCRT, and ACT, severe (grade 3 or higher) hematologic toxicity was experienced by $29.3 \%$ (12/41), $14.5 \%(12 / 83)$, and $58.3 \%$ (7/12) of patients, respectively. Grade 4 hematologic toxicity occurred in 12.2\% (5/41), 2.4\% (2/83), and $16.7 \%(2 / 12)$ of patients during NCT, CCRT, and ACT, respectively. Of the 83 patients, 48 patients (57.8\%) experienced severe non-hematologic toxicity during CCRT. The most common event was grade 3 mucositis of the oral cavity or pharynx, which significantly decreased from $34.9 \%$ to $22.9 \%$ with the use of NCT $(p=0.036)$. Grade 4 nonhematologic toxicity was not observed during CCRT.

The use of NCT or ACT resulted in significantly higher overall 
Table 3. Predictors of disease outcomes in univariate analysis

\begin{tabular}{|c|c|c|c|c|c|c|c|c|c|c|}
\hline \multirow[b]{2}{*}{ Variable } & \multicolumn{2}{|c|}{$\mathrm{LC}$} & \multicolumn{2}{|c|}{$\mathrm{RC}$} & \multicolumn{2}{|c|}{ DMFS } & \multicolumn{2}{|c|}{ DFS } & \multicolumn{2}{|c|}{ OS } \\
\hline & $\begin{array}{c}\text { 5-yr rate } \\
(\%)\end{array}$ & $p$-value & $\begin{array}{c}5-y r \text { rate } \\
(\%)\end{array}$ & $p$-value & $\begin{array}{c}5-y r \text { rate } \\
(\%)\end{array}$ & $p$-value & $\begin{array}{c}5-y r \text { rate } \\
(\%)\end{array}$ & p-value & $\begin{array}{c}5-y r \text { rate } \\
(\%)\end{array}$ & $p$-value \\
\hline Overall & 94.7 & & 89.3 & & 77.8 & & 64.1 & & 81.8 & \\
\hline \multicolumn{11}{|l|}{ Age (yr) } \\
\hline$<50$ & 95.7 & & 92.9 & & 80.4 & & 69.9 & & 88.2 & \\
\hline$\geq 50$ & 94.0 & 0.385 & 84.8 & 0.281 & 74.2 & 0.939 & 65.7 & 0.734 & 73.6 & 0.103 \\
\hline \multicolumn{11}{|l|}{ Sex } \\
\hline Male & 97.9 & & 90.1 & & 72.2 & & 68.4 & & 86.5 & \\
\hline Female & 87.4 & 0.185 & 87.8 & 0.654 & 91.6 & 0.092 & 67.2 & 0.892 & 72.6 & 0.491 \\
\hline \multicolumn{11}{|l|}{ Histology } \\
\hline WHO I-Ila & 100 & & 90.9 & & 82.3 & & 74.5 & & 80.0 & \\
\hline WHO IIb & 92.7 & 0.279 & 88.6 & 0.809 & 76.0 & 0.713 & 65.4 & 0.598 & 83.0 & 0.767 \\
\hline \multicolumn{11}{|c|}{ T classification } \\
\hline $\mathrm{T} 1-\mathrm{T} 2$ & 90.6 & & 84.0 & & 75.0 & & 63.2 & & 84.6 & \\
\hline T3-T4 & 97.9 & 0.396 & 93.2 & 0.231 & 79.9 & 0.528 & 71.8 & 0.401 & 79.5 & 0.668 \\
\hline \multicolumn{11}{|c|}{$\mathrm{N}$ classification } \\
\hline NO & 100 & & 100 & & 87.5 & & 87.5 & & 100 & \\
\hline N1-2 & 98.1 & & 94.0 & & 85.7 & & 78.6 & & 83.7 & \\
\hline N3b & 85.0 & 0.262 & 72.2 & 0.032 & 55.8 & 0.005 & 36.2 & $<0.001$ & 72.0 & 0.115 \\
\hline \multicolumn{11}{|l|}{ Stage group } \\
\hline III & 97.2 & & 94.5 & & 93.7 & & 85.8 & & 82.3 & \\
\hline IVA-IVB & 92.6 & 0.683 & 84.9 & 0.247 & 66.0 & 0.006 & 54.5 & 0.009 & 81.5 & 0.946 \\
\hline \multicolumn{11}{|l|}{ NCT } \\
\hline$(-)$ & 100 & & 92.1 & & 81.4 & & 76.8 & & 85.4 & \\
\hline$(+)$ & 90.5 & 0.106 & 86.6 & 0.472 & 75.7 & 0.707 & 61.3 & 0.222 & 80.5 & 0.725 \\
\hline \multicolumn{11}{|l|}{ NCT or ACT } \\
\hline$(-)$ & 100 & & 88.8 & & 89.4 & & 82.5 & & 82.9 & \\
\hline$(+)$ & 92.4 & 0.232 & 89.7 & 0.858 & 73.1 & 0.222 & 62.1 & 0.184 & 82.2 & 0.884 \\
\hline
\end{tabular}

LC, local control; RC, regional control; DMFS, distant metastasis-free survival; DFS, disease-free survival; OS, overall survival; WHO, World Health Organization; NCT, neoadjuvant chemotherapy; ACT, adjuvant chemotherapy.

a) Log-rank test.

Table 4. Treatment-related toxicity profiles during each treatment course

\begin{tabular}{|c|c|c|c|c|c|c|}
\hline \multirow{2}{*}{ Toxicities } & \multicolumn{2}{|c|}{$\mathrm{NCT}(\mathrm{n}=41)$} & \multicolumn{2}{|c|}{ CCRT $(n=83)$} & \multicolumn{2}{|c|}{$\mathrm{ACT}(\mathrm{n}=12)$} \\
\hline & Grade 3 & Grade 4 & Grade 3 & Grade 4 & Grade 3 & Grade 4 \\
\hline Hematologic & \multicolumn{2}{|c|}{$12(29.2)$} & \multicolumn{2}{|c|}{$12(14.5)$} & \multicolumn{2}{|c|}{7 (58.3) } \\
\hline Anemia & $0(0)$ & $0(0)$ & $3(3.6)$ & $0(0)$ & $0(0)$ & $0(0)$ \\
\hline Neutropenia & 7 (17.1) & $5(12.2)$ & $7(8.4)$ & $1(1.2)$ & $5(41.7)$ & 2 (16.7) \\
\hline Thrombocytopenia & $0(0)$ & $0(0)$ & $3(3.6)$ & $1(1.2)$ & 0 & $0(0)$ \\
\hline Non-hematologic & \multicolumn{2}{|c|}{$1(2.4)$} & \multicolumn{2}{|c|}{$48(57.8)$} & \multicolumn{2}{|c|}{$1(8.3)$} \\
\hline Mucositis & $1(2.4)$ & $0(0)$ & $42(50.6)$ & $0(0)$ & $0(0)$ & $0(0)$ \\
\hline Dermatitis & $0(0)$ & $0(0)$ & $5(6.0)$ & $0(0)$ & $0(0)$ & $0(0)$ \\
\hline Nausea & $0(0)$ & $0(0)$ & $4(4.8)$ & $0(0)$ & $0(0)$ & $0(0)$ \\
\hline Weight Loss & $0(0)$ & $0(0)$ & $1(1.2)$ & $0(0)$ & $0(0)$ & $0(0)$ \\
\hline Infection & $0(0)$ & $0(0)$ & $0(0)$ & $0(0)$ & $0(0)$ & 1 (8.3) \\
\hline
\end{tabular}

Values are presented as number (\%).

NCT, neoadjuvant chemotherapy; CCRT, concurrent chemoradiation; ACT, adjuvant chemotherapy. 
risk of severe hematologic toxicity at some point during the treatment course compared to patients treated by CCRT alone $(30.1 \%$ vs. $6.0 \%$, respectively; $p=0.005)$. The use of NCT also showed a non-significant trend towards increased severe hematologic toxicity during CCRT (10.8\% vs. 3.6\%, respectively; $p=0.055$ ). However, severe non-hematologic toxicity during CCRT occurred more often in patients treated without NCT compared to those treated with NCT (34.9\% vs. $22.9 \%$, respectively; $p=0.035$ ). In a comparison of patients treated with CCRT alone and CCRT plus ACT, ACT increased the overall risk of severe hematologic toxicity at some point during the treatment course ( $16.7 \%$ vs. $11.9 \%$, respectively; $p=0.007$ ).

Severity of xerostomia was recorded in the medical records according to the RTOG scale. Severe xerostomia did not occur during RT or follow-up. The rate of clinically assessed xerostomia of any grade was 79.5\% (66/83), 85.5\% (65/76), and $76.9 \%$ (50/65) on the last week of RT, one year post-RT, and two years post-RT, respectively. However, the rate of grade 2 xerostomia decreased over time and was only $32.5 \%$ (27/83), $14.5 \%(11 / 76)$, and $6.2 \%(4 / 65)$ on the last week of RT, one year post-RT, and two years post-RT, respectively.

\section{Subgroup analysis I: NCT plus CCRT vs. CCRT alone}

To validate the role of NCT in addition to CCRT, we performed a subgroup analysis with 71 patients. We compared 41 patients treated with NCT followed by CCRT and 30 patients treated with CCRT alone. The median follow-up for survivors was 63.4 months (range, 6.0 to 123.7 months) for patients treated with NCT followed by CCRT and 38.6 months (range, 6.3 to 102.9 months) for patients treated with CCRT alone. Overall, NCT

A

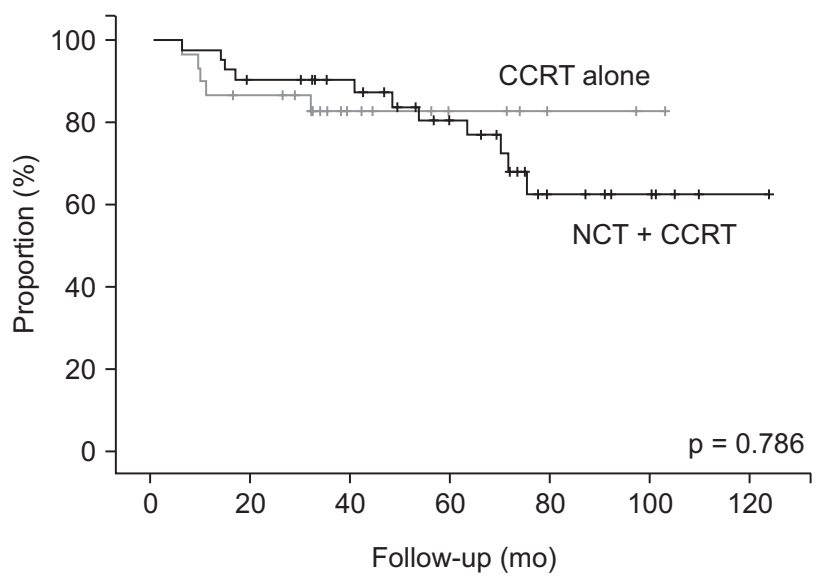

did not improve outcomes for any clinical endpoint (Fig. 2). Moreover, although not statistically significant, CCRT alone resulted in better outcomes for every endpoints at five years. However, the $N$ stage $(p=0.007)$ and AJCC stage $(p=0.035)$ were more advanced in the NCT plus CCRT group, and both remained significant prognostic factors for DMFS and DFS in a univariate analysis of the 71 patients in the subgroup analysis. Therefore, to adjust for the $\mathrm{N}$ stage and AJCC stage in DMFS and DFS, we performed further analysis with a Cox proportional hazard model with two variables simultaneously: N stage with NCT use and AJCC stage with NCT use (results not shown). For DMFS, the $\mathrm{N}$ stage remained significant $(\mathrm{p}=0.017)$, while NCT did not affect outcomes $(p=0.790)$. Similarly, N stage $(p=0.006)$ and AJCC stage $(p=0.026)$ both remained significant for DFS, while NCT did not affect results $(p=0.742$ and $p=0.461$, respectively). However, the significance of AJCC stage on DMFS was diminished $(p=0.952)$ with the use of NCT. The use of NCT prior to CCRT led to increased risk of severe hematologic toxicity during the treatment course (25.4\% vs. $7.0 \% ; p=0.015)$. The details of patient characteristics and survival outcomes of the subgroup analysis are shown on Tables 5 and 6 , respectively.

\section{Subgroup analysis II: CCRT alone vs. CCRT plus ACT}

Similar to subgroup analysis I, 30 patients treated by CCRT alone were compared to 12 patients treated with CCRT plus ACT. Comparing patients treated with CCRT alone with those treated with CCRT plus ACT, the 5-year DMFS, DFS, and OS rates were $89.4 \%$ vs. $66.7 \%(p=0.151), 82.5 \%$ vs. $66.7 \%$ ( $p$ $=0.440)$, and $82.9 \%$ vs. $91.7 \%(p=0.849)$, respectively. The

B

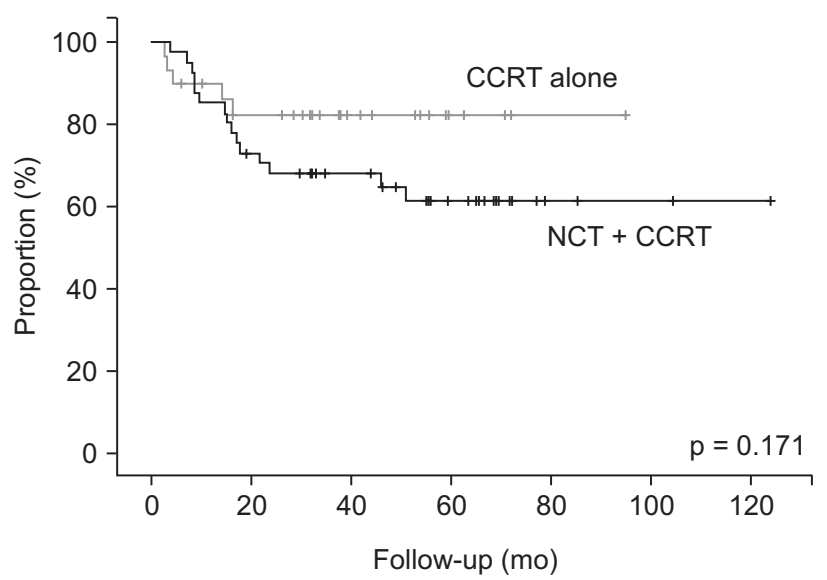

Fig. 2. Kaplan-Meier plots of overall survival (A) and disease-free survival (B) comparing patients treated by concurrent chemoradiation (CCRT) with or without neoadjuvant chemotherapy (NCT). 
ROJ Radiation Oncology Journal

Table 5. Patient characteristics from subgroup analysis I

\begin{tabular}{lrrr}
\hline \multicolumn{1}{c}{ Variable } & CCRT alone & NCT + CCRT & p-value \\
\hline Age & $52.5(29-77)$ & $48(21-71)$ & 0.091 \\
Sex & & & \\
$\quad$ Male & $19(63.3)$ & $30(73.2)$ & \\
$\quad$ Female & $11(36.7)$ & $11(26.8)$ & 0.376 \\
Histology & & & \\
$\quad$ WHO I-Ila & $8(26.7)$ & $11(26.8)$ & \\
$\quad$ WHO Ilb & $22(73.3)$ & $30(73.2)$ & 0.988 \\
T classification & & & \\
$\quad$ T1-T2 & $8(26.7)$ & $21(51.2)$ & \\
$\quad$ T3-T4 & $22(77.7)$ & $20(48.8)$ & 0.223 \\
N classification & & & \\
$\quad$ NO & $6(20.0)$ & $0(0)$ & \\
$\quad$ N1-2 & $17(56.7)$ & $26(63.4)$ & \\
$\quad$ N3 & $7(23.3)$ & $15(36.6)$ & 0.007 \\
Stage group & & & \\
III & $17(56.7)$ & $13(31.7)$ & \\
$\quad$ IVA-IVB & $13(43.3)$ & $28(68.3)$ & 0.035 \\
\hline
\end{tabular}

Values are presented as median (range) or number of patients (\%). CCRT, concurrent chemoradiation; NCT, neoadjuvant chemotherapy.

$\mathrm{LC}$ and $\mathrm{RC}$ rates could not be analyzed by the log-rank test because all cases were censored. Severe hematologic toxicity significantly increased with ACT use (58.3\% vs. $16.7 \% ; p=$ 0.007).

\section{Discussion and Conclusion}

In our study, treatment of LA-NPC by CCRT using weekly cisplatin chemotherapy and IMRT (67.5 Gy in 30 fractions to the primary tumor) resulted in excellent locoregional disease control and survival. When used in addition to CCRT, neither NCT nor ACT improved LC, RC, DMFS, DFS, or OS. However, the number of severe hematologic events significantly increased with NCT or ACT use, although most were manageable and uncomplicated.

Historical data show that only about half of LA-NPC patients survive for five years after treatment with RT alone $[3,4]$. Therefore, issues of adding chemotherapy to RT emerged, with Al-Sarraf et al. [5] first demonstrating the benefit of CCRT plus ACT over RT alone in LA-NPC patients in a phase III randomized trial. However, the study was criticized for the poor OS in the arm treated with RT alone, the discrepancy of histological proportions with endemic areas, and poor compliance to ACT. Therefore, similar phase III randomized studies of mainly LANPC from endemic areas were soon reported. Comparing CCRT with or without ACT to RT alone, most of them demonstrated
CCRT with IMRT in stage III-IV nasopharyngeal carcinoma

Table 6. Actuarial rates (\%) of clinical outcomes at 5 years from subgroup analysis

\begin{tabular}{lccc}
\hline & \multicolumn{3}{c}{ Stage III-IVB } \\
\cline { 2 - 4 } & NCT + CCRT & CCRT alone & p-value ${ }^{\text {a) }}$ \\
\hline OS & 80.5 & 82.9 & 0.786 \\
PFS & 61.3 & 69.5 & 0.728 \\
LC & 90.5 & 100 & 0.178 \\
RC & 86.6 & 88.8 & 0.844 \\
DMF & 75.7 & 89.4 & 0.320 \\
\hline
\end{tabular}

NCT, neoadjuvant chemotherapy; CCRT, concurrent chemoradiation; OS, overall survival; PFS, progression-free survival; LC, local control; $\mathrm{RC}$, regional control; DMFS, distant metastasis-free survival.

a) Log-rank test.

significant OS benefit of 7\%-23\% which resulted in actual OS rates of $90 \%$ at $2-5$ years by adding chemotherapy [6-10]. The 2 -year OS of $89.1 \%$ (vs. 85\%-100\% $[6,7,10])$ and 5 -year OS of $81.8 \%$ (vs. $63 \%-72 \%[5,8,9]$ ) from our study were comparable or superior to those of the chemoradiation arm from the 5 studies which exclusively counted in stage III-IVB patients.

Two meta-analyses also agree with this finding $[20,21]$, reporting an OS benefit of 4\%-6\% by adding chemotherapy to RT alone when chemotherapy is added concurrently, but not sequentially. Recently, a phase III randomized study [11] comparing CCRT alone to CCRT plus ACT failed to demonstrate a survival benefit with ACT use. Two-year failure-free survival was $84 \%$ and $86 \%$ in the CCRT alone arm and the CCRT plus ACT arm, respectively, and were not statistically different ( $p$ $=0.130$ ). There was also no improvement in the 2-year OS. Similar results were shown in our subgroup analysis, despite much better compliance to ACT compared to historical completion rates of 52\%-63\% [5-7,11] after CCRT, with 92\% of patients completing three or more cycles. However, the authors refrained from making any further conclusions from this subgroup analysis since the number of patients treated with CCRT plus ACT was too small.

Since tolerance of ACT after CCRT is very poor due to the substantial toxicity resulting from CCRT, another strategy of adding NCT to CCRT can be considered to improve compliance and, eventually, the efficacy of chemotherapy. Encouraging phase II studies have revealed that NCT is well-tolerated [22]. More than $85 \%$ of patients completed the planned three cycles of NCT. Compliance to NCT in our study was also excellent, with 93\% completing all three planned cycles. The early introduction of full dose cytotoxic chemotherapy may have an advantage in early eradication of micrometastasis and therefore eventually reduce distant metastasis. Indeed, 
according to a meta-analysis [20], NCT did reduce distant metastasis when added to RT alone. However, it is unclear whether this benefit would still be present when NCT is added to CCRT. The 5-year DMFS rate, which should improve with micrometastasis eradication, was $76 \%$ in patients receiving NCT. This was not different from that of patients treated by CCRT alone. One interesting finding from our subgroup analysis was that the significance of AJCC stage for DMFS, consistent throughout the study, diminished with NCT use. This might mean that, compared to stage III patients, stage IVA-IVB patients are exposed to significantly higher risks of developing distant metastasis. Early introduction of full dose chemotherapy by NCT might potentially overcome this difference. The use of NCT also may allow a smaller high-dose RT field by shrinking the primary or nodal tumor burden. According to our study, severe RT-related toxicity, mainly mucositis of the oral cavity or pharynx, significantly decreased with NCT use. This might be attributable to tumor shrinkage since the GTV for IMRT was delineated as the postchemotherapy tumor volume in patients treated with NCT. Nevertheless, there is a significant risk for severe hematologic toxicity when using NCT compared to CCRT alone. The risks and benefits should be carefully considered based on each individual patient.

So far, there are no reported phase III randomized trial result solely comparing NCT plus CCRT with CCRT alone. Only two randomized phase II trials have been reported, with contradicting results. Hui et al. [18] reported a positive impact on survival in 65 LA-NPC patients with NCT using a docetaxel/ cisplatin regimen in addition to CCRT with weekly cisplatin. The dose and schedule of the docetaxel/cisplatin regimen were the same as the one used here, but Hui et al. [18] applied a median of only two NCT cycles. Despite the lower number of NCT cycles, there was a significant improvement of $26.4 \%$ in the 3 -year OS ( $p=0.012)$. There was no difference in the risk of acute toxicities during CCRT. In contrast to Hui et al. [18], the Hellenic Cooperative Oncology Group (HeCOG) failed to improve response rates and survival in 141 LA-NPC patients with a NCT regimen of cisplatin, epirubicin, and paclitaxel in addition to CCRT with weekly cisplatin [19]. The 3-year progression-free survival was $64.5 \%$ vs. $63.5 \%(p=0.708)$ for the NCT plus CCRT and CCRT alone arms, respectively. Three-year OS was $66.6 \%$ vs. $71.8 \%$, respectively ( $p=0.652$ ). Although the $\mathrm{HeCOG}$ trial can be criticized for the inclusion of stage IIB patients, they only accounted for less than 25\%. However, the 3-year OS rates for CCRT alone, $67.7 \%$ in the study of Hui et al. [18] and 71.8\% from HeCOG study, were inferior to the 5 -year OS rate of $82.9 \%$ from our study. This finding makes the interpretation more complex and we eagerly await the unpublished data from four phase III randomized trials. Three different NCT regimens, applied in addition to cisplatinbased CCRT, are being evaluated in these four trials; mitomycin C, epirubicin, cisplatin, 5-fluorouracil, and leucovorin from Taiwan (NCT00201396); gemcitabine, carboplatin, and paclitaxel from Singapore (NCT00997906); and docetaxel/5-fluorouracil/ cisplatin in two trials from China (NCT01245959) and France (NCT00828386). Except the Chinese trial, which adopted a triweekly concurrent cisplatin regimen during CCRT, the trials use a weekly cisplatin schedule. Only the Singapore trial strictly requires IMRT as the RT modality. The results of these ongoing trials are expected to define the role of NCT in addition to CCRT.

Regarding RT, IMRT is already widely acknowledged as the standard modality in HEtN cancer, with excellent locoregional disease control at reduced toxicity rates [23]. Large-scale IMRT series mainly including LA-NPC patients have reported $L C$ rates exceeding $90 \%$ at 2-5 years with various dose-fractionation schemes [24-27]. Lin et al. [24] reported grade 2 xerostomia rates of $63.8 \%$ and $7.8 \%$ at one and two years, respectively. Wong et al. [26] also reported a late grade 2 xerostomia rate of $2.3 \%$, although the timing of evaluation was unavailable. The largest study, with 512 stage III-IV patients from Sun Yat-Sen University of China [27], lacked information on xerostomia. LC with IMRT at our institution using a dose of $67.5 \mathrm{~Gy}$ in 30 fractions prescribed to the primary tumor was comparable or even superior to those from the studies mentioned above. Grade 2 xerostomia rates at one-year and two-year post-RT were $14.5 \%$ and $6.2 \%$, respectively. These were lower than the rates from the study of Lin et al. [24] but not directly comparable with those from Wong et al. [26] However, it is obvious that these numbers are far more acceptable than those from the era of two-dimensional RT [28] and threedimensional conformal RT. Nevertheless, dose escalation up to 81 Gy failed to improve outcomes [29]. Improving LC to a level higher than the current rate seems to be quite difficult and achieving approximately 100\% LC will likely require much time. Testing unevaluated systemic agents would be a much more reasonable strategy for now.

Several limitations exist in our study, including the retrospective nature of the study, the small number of studied patients, uneven distributions in follow-up duration and patient characteristics between groups in the subgroup 
ROJ Radiation Oncology Journal

analysis, and the use of a heterogeneous NCT regimen in the NCT plus CCRT cohort. However, almost every patient completed the entire planned treatment course and the CCRT regimen was absolutely homogeneous. Therefore, this study should be differentiated from other retrospective series.

In conclusion, we observed excellent LC and survival outcomes for the 83 LA-NPC patients treated by IMRT with $67.5 \mathrm{~Gy}$ in 30 fractions and concurrent weekly cisplatin chemotherapy at our institution. Compliance for the CCRT, NCT, and ACT regimens was excellent. Although NCT usage failed to provide improvement in survival while significantly increasing the risk of severe hematologic toxicity, it provided some benefit in reducing the risk of severe RT-related mucositis during CCRT and demonstrated potential benefit of improving DMFS for stage IV patients. Due to the lack of evidence of OS benefit, risk of increased toxicity, higher patient costs, delay of local therapy, and prolongation of treatment duration, NCT should be carefully administered in LA-NPC patients, especially for stage IVA-IVB patients. Ongoing randomized studies are expected to define the role of NCT and the subset of patients who would benefit from the treatment.

\section{Conflict of Interest}

No potential conflict of interest relevant to this article was reported.

\section{Acknowledgments}

This study was supported by grants from the Korean Health Technology R\&D Project, Ministry of Health \& Welfare, Republic of Korea (HI14C3459) and by National R\&D Program through the Dong-nam Institute of Radiological \& Medical Sciences (DIRAMS) funded by the Ministry of Education, Science and Technology (50595-2014).

\section{References}

1. Vokes EE, Liebowitz DN, Weichselbaum RR. Nasopharyngeal carcinoma. Lancet 1997;350:1087-91.

2. Ali H, Al-Sarraf M. Chemotherapy in advanced nasopharyngeal cancer. Oncology (Williston Park) 2000;14:1223-30.

3. Geara FB, Sanguineti G, Tucker $S L$, et al. Carcinoma of the nasopharynx treated by radiotherapy alone: determinants of distant metastasis and survival. Radiother Oncol 1997:43:5361.

4. Hong S, Wu HG, Chie EK, et al. Neoadjuvant chemotherapy

\section{CCRT with IMRT in stage III-IV nasopharyngeal carcinoma}

and radiation therapy compared with radiation therapy alone in advanced nasopharyngeal carcinoma. Int J Radiat Oncol Biol Phys 1999;45:901-5.

5. Al-Sarraf M, LeBlanc M, Giri PG, et al. Chemoradiotherapy versus radiotherapy in patients with advanced nasopharyngeal cancer: phase III randomized Intergroup study 0099. J Clin Oncol 1998;16:1310-7.

6. Wee J, Tan EH, Tai BC, et al. Randomized trial of radiotherapy versus concurrent chemoradiotherapy followed by adjuvant chemotherapy in patients with American Joint Committee on Cancer/International Union against cancer stage III and IV nasopharyngeal cancer of the endemic variety. J Clin Oncol 2005;23:6730-8.

7. Chen Y, Liu MZ, Liang SB, et al. Preliminary results of a prospective randomized trial comparing concurrent chemoradiotherapy plus adjuvant chemotherapy with radiotherapy alone in patients with locoregionally advanced nasopharyngeal carcinoma in endemic regions of china. Int J Radiat Oncol Biol Phys 2008;71:1356-64.

8. Lin JC, Jan JS, Hsu CY, Liang WM, Jiang RS, Wang WY. Phase III study of concurrent chemoradiotherapy versus radiotherapy alone for advanced nasopharyngeal carcinoma: positive effect on overall and progression-free survival. J Clin Oncol 2003;21: $631-7$

9. Chan AT, Leung SF, Ngan RK, et al. Overall survival after concurrent cisplatin-radiotherapy compared with radiotherapy alone in locoregionally advanced nasopharyngeal carcinoma. J Natl Cancer Inst 2005;97:536-9.

10. Zhang L, Zhao C, Peng PJ, et al. Phase III study comparing standard radiotherapy with or without weekly oxaliplatin in treatment of locoregionally advanced nasopharyngeal carcinoma: preliminary results. J Clin Oncol 2005;23:8461-8.

11. Chen L, Hu CS, Chen XZ, et al. Concurrent chemoradiotherapy plus adjuvant chemotherapy versus concurrent chemoradiotherapy alone in patients with locoregionally advanced nasopharyngeal carcinoma: a phase 3 multicentre randomised controlled trial. Lancet Oncol 2012;13:163-71.

12. National Comprehensive Cancer Network. NCCN Clinical Practice Guidelines in Oncology (NCCN Guidelines): head and neck cancers v2.2014 [Internet]. Fort Washington, PA: National Comprehensive Cancer Network; c2015 [cited 2015 Jan 8]. Available from: http://www.nccn.org/professionals/physician_ gls/pdf/head-and-neck.pdf.

13. Chan AT, Teo PM, Leung TW, et al. A prospective randomized study of chemotherapy adjunctive to definitive radiotherapy in advanced nasopharyngeal carcinoma. Int J Radiat Oncol Biol Phys 1995;33:569-77.

14. Ma J, Mai HQ, Hong MH, et al. Results of a prospective randomized trial comparing neoadjuvant chemotherapy plus radiotherapy with radiotherapy alone in patients with locoregionally advanced nasopharyngeal carcinoma. J Clin 
Oncol 2001;19:1350-7.

15. Chua DT, Sham JS, Choy D, et al. Preliminary report of the Asian-Oceanian Clinical Oncology Association randomized trial comparing cisplatin and epirubicin followed by radiotherapy versus radiotherapy alone in the treatment of patients with locoregionally advanced nasopharyngeal carcinoma. Cancer 1998;83:2270-83.

16. Hareyama $M$, Sakata $K$, Shirato $H$, et al. A prospective, randomized trial comparing neoadjuvant chemotherapy with radiotherapy alone in patients with advanced nasopharyngeal carcinoma. Cancer 2002;94:2217-23.

17. Chua DT, Ma J, Sham JS, et al. Long-term survival after cisplatin-based induction chemotherapy and radiotherapy for nasopharyngeal carcinoma: a pooled data analysis of two phase III trials. J Clin Oncol 2005;23:1118-24.

18. Hui EP, Ma BB, Leung SF, et al. Randomized phase II trial of concurrent cisplatin-radiotherapy with or without neoadjuvant docetaxel and cisplatin in advanced nasopharyngeal carcinoma. J Clin Oncol 2009;27:242-9.

19. Fountzilas G, Ciuleanu E, Bobos M, et al. Induction chemotherapy followed by concomitant radiotherapy and weekly cisplatin versus the same concomitant chemoradiotherapy in patients with nasopharyngeal carcinoma: a randomized phase II study conducted by the Hellenic Cooperative Oncology Group (HeCOG) with biomarker evaluation. Ann Oncol 2012;23:427-35.

20. Langendijk JA, Leemans CR, Buter J, Berkhof J, Slotman BJ. The additional value of chemotherapy to radiotherapy in locally advanced nasopharyngeal carcinoma: a meta-analysis of the published literature. J Clin Oncol 2004;22:4604-12.

21. Baujat B, Audry H, Bourhis J, et al. Chemotherapy in locally advanced nasopharyngeal carcinoma: an individual patient data meta-analysis of eight randomized trials and 1753 patients. Int J Radiat Oncol Biol Phys 2006;64:47-56.
22. Lee AW, Ng WT, Chan OS, Sze HC. If concurrent-adjuvant chemoradiotherapy is beneficial for locoregionally advanced nasopharyngeal carcinoma, would changing the sequence to induction-concurrent achieve better outcome? J Radiat Oncol 2012;1:107-15

23. Veldeman L, Madani I, Hulstaert F, De Meerleer G, Mareel M, De Neve W. Evidence behind use of intensity-modulated radiotherapy: a systematic review of comparative clinical studies. Lancet Oncol 2008;9:367-75.

24. Lin S, Pan J, Han L, Zhang $X$, Liao X, Lu JJ. Nasopharyngeal carcinoma treated with reduced-volume intensity-modulated radiation therapy: report on the 3-year outcome of a prospective series. Int J Radiat Oncol Biol Phys 2009;75:10718.

25. Tham IW, Hee SW, Yeo RM, et al. Treatment of nasopharyngeal carcinoma using intensity-modulated radiotherapy-the national cancer centre singapore experience. Int J Radiat Oncol Biol Phys 2009;75:1481-6.

26. Wong FC, Ng AW, Lee VH, et al. Whole-field simultaneous integrated-boost intensity-modulated radiotherapy for patients with nasopharyngeal carcinoma. Int J Radiat Oncol Biol Phys 2010;76:138-45.

27. Lai SZ, Li WF, Chen L, et al. How does intensity-modulated radiotherapy versus conventional two-dimensional radiotherapy influence the treatment results in nasopharyngeal carcinoma patients? Int J Radiat Oncol Biol Phys 2011;80:661-8.

28. Lee NK, Park YJ, Yang DS, Yoon WS, Lee S, Kim CY. Long-term results of 2-dimensional radiation therapy in patients with nasopharyngeal cancer. J Korean Soc Ther Radiol Oncol 2010; 28:193-204.

29. Yeh SA, Huang YJ. Dose escalation for patients with locally advanced nasopharyngeal carcinoma treated with radiotherapy alone. Am J Clin Oncol 2007;30:401-5. 